\title{
Central and Peripheral Axon Branches from One Neuron Are Guided Differentially by Semaphorin3D and Transient Axonal Glycoprotein-1
}

\author{
Yan Liu and Mary C. Halloran \\ Departments of Zoology and Anatomy, University of Wisconsin, Madison, Wisconsin 53706
}

\begin{abstract}
For multiple axons from one neuron to extend in different directions to unique targets, the growth cones of each axon must have distinct responses to guidance cues. However, the mechanisms by which separate axon branches are guided along different pathways are mainly unknown. Zebrafish Rohon-Beard (R-B) sensory neurons extend central axon branches in the spinal cord and peripheral axons to the epidermis. To investigate the differential guidance mechanisms of the central versus peripheral R-B axon branches, we used live-growth cone imaging in vivo combined with manipulation of individual guidance molecules. We show that a semaphorin expressed at the dorsal spinal cord midline, Semaphorin3D (Sema3D), may act to repel the peripheral axons out of the spinal cord. Sema3D knock-down reduces the number of peripheral axons. Remarkably, Sema3D ectopic expression repels and induces branching of peripheral axons in vivo but has no effect on central axons from the same neurons. Conversely, central axons require a growth-promoting molecule, transient axonal glycoprotein-1 (TAG-1), to advance, whereas peripheral axons do not. After TAG-1 knock-down, central growth cones display extensive protrusive activity but make little forward advance. TAG-1 knock-down has no effect on the motility or advance of peripheral growth cones. These experiments show how Sema3D and TAG-1 regulate the motility and behavior of growth cones extending in their natural in vivo environment and demonstrate that two different axon branches from one neuron respond differently to guidance cues in vivo.
\end{abstract}

Key words: axon guidance; sensory neurons; Rohon-Beard neurons; semaphorin; zebrafish; Ig superfamily

\section{Introduction}

Neurons have complex and distinct patterns of axonal and dendritic arbors that serve specific functions. During development, these arbors form by directed outgrowth of neurites along specific pathways. For multiple neurites from one neuron to extend in different directions to unique targets, the growth cones of each neurite must have distinct responses to guidance cues. Recent studies have begun to reveal mechanisms by which axons versus dendrites are guided differently, often by the same guidance cue (Polleux et al., 2000; Furrer et al., 2003; Kim and Chiba, 2004). For example, the axons and dendrites of cortical neurons extend in opposite directions and respond oppositely to the secreted guidance cue Semaphorin3A (Sema3A) (Polleux et al., 2000). However, the mechanisms by which two axon branches from one cell are guided differentially along separate pathways are understood poorly. Mouse knock-outs of Sema3A or its receptors PlexinA3/A4 show excessive growth of peripheral dorsal root ganglion axons, whereas the central projections appear grossly

\footnotetext{
Received June 30, 2005; revised 0ct. 3, 2005; accepted 0ct. 3, 2005.

This work was supported by National Science Foundation Grant IBN 0110654 and National Institutes of Health Grant NS42228 to M.C.H. We thank Bill Bement, Tim Gomez, and lain Drummond for discussion and comments on this manuscript and Claudia Stuermer for the TAG-1 antibody.

Correspondence should be addressed to Mary C. Halloran, Department of Zoology, University of Wisconsin, 1117 West Johnson Street, Madison, WI 53706. E-mail: mchalloran@wisc.edu.

Y. Liu's present address: Renal Unit, Massachusetts General Hospital, 149 13th Street, Charlestown, MA 02129. E-mail: yliu@receptor.mgh.harvard.edu.

DOI:10.1523/JNEUROSCI.2710-05.2005

Copyright $\odot 2005$ Society for Neuroscience $\quad 0270-6474 / 05 / 2510556-08 \$ 15.00 / 0$
}

normal (Taniguchi et al., 1997; Yaron et al., 2005). However, a different Sema3A knock-out mouse showed aberrant growth of central projections (Behar et al., 1996), suggesting that Sema3A affects both axon branches. An Ig superfamily molecule, Down syndrome cell adhesion molecule (DSCAM), controls the initial separation of sister branches of bifurcating growth cones in Drosophila (Wang et al., 2002), but it is not known how the branches respond differently to subsequent guidance cues.

We investigated mechanisms of differential axon guidance in zebrafish Rohon-Beard (R-B) sensory neurons, which have central and peripheral axon branches with distinct projection pathways, behaviors, and targets, although the mechanisms by which they are guided differentially are not understood (Clarke et al., 1984; Bernhardt et al., 1990). Recent work has shown that the Lin1, Islet-1, mechanosensory abnormal-3 (LIM) homeodomain transcription factor Islet 2 (Isl2), and its cofactor LIM are required for peripheral axons to form (Segawa et al., 2001; Becker et al., 2002), although to date, the downstream genes controlling peripheral axon formation are not known. PlexinA4, a guidance receptor on $\mathrm{R}-\mathrm{B}$ cells, plays a role in the secondary arborization of peripheral axons in the epidermis but not in their initial formation or directed outgrowth (Miyashita et al., 2004).

The clarity and simplicity of the zebrafish embryo allows us to visualize the living behavior of individual R-B axon branches as they extend in their natural environment. We have used live imaging together with molecular manipulations to show that two guidance molecules differentially affect the motility and guidance 

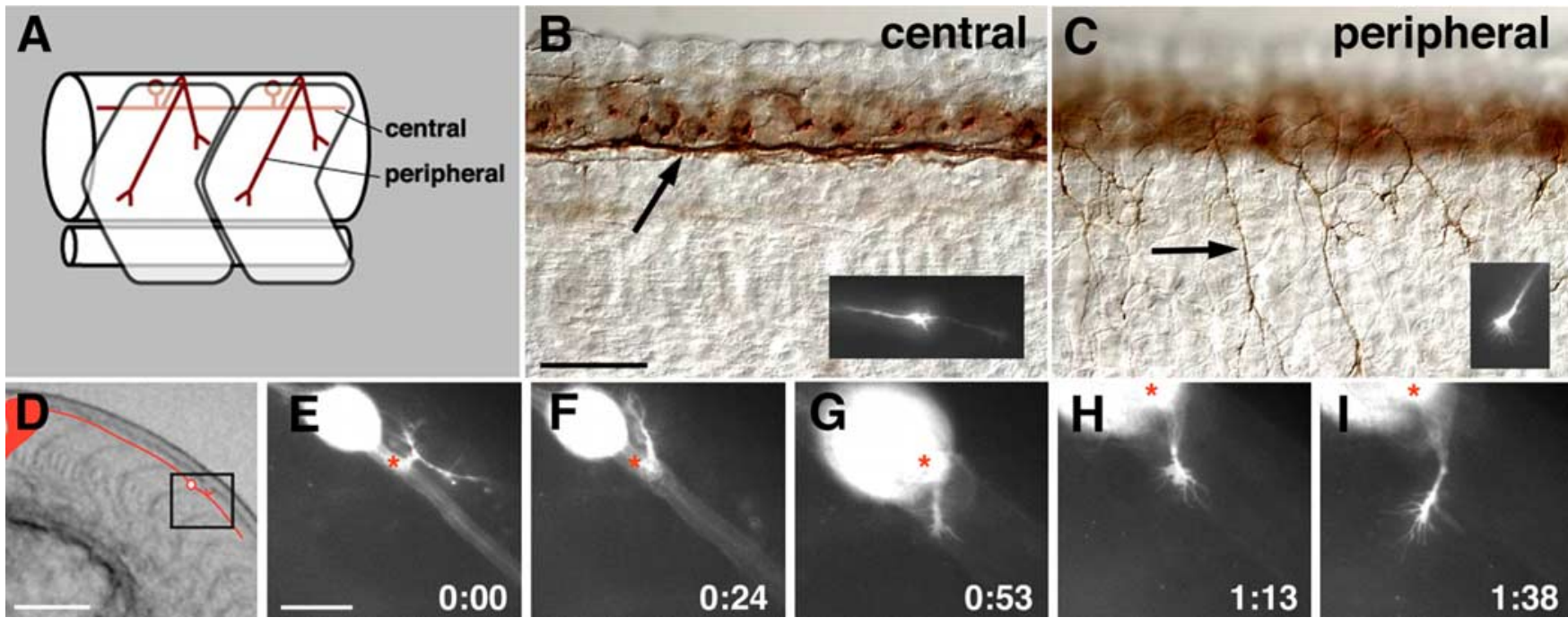

Figure 1. The peripheral R-B axon branches off the central axon. $A$, Schematic lateral view of trunk showing R-B axon pathways. $B$, C, Lateral views of $22 \mathrm{hpf} Z \mathrm{~N}-12$-labeled embryo showing central axons in spinal cord focal plane ( $\boldsymbol{B}$, arrow) and peripheral axons in epidermal focal plane ( $\boldsymbol{C}$, arrow). Insets show typical Dil-labeled living growth cones. $\boldsymbol{D}$, The $21 \mathrm{hpf}$ embryo showing position of Dil injection site (red) and labeled R-B axon and cell body. The box indicates the region shown in $\boldsymbol{E}-\boldsymbol{I}$. $\boldsymbol{E}-\boldsymbol{I}$, Images from time-lapse sequence of peripheral R-B branch emerging. The red asterisk marks fixed reference point. Scale bars: $\boldsymbol{B}, 50 \mu \mathrm{m} ; \boldsymbol{D}, 100 \mu \mathrm{m} ; \boldsymbol{E}, 20 \mu \mathrm{m}$.

of the central versus peripheral growth cones. Semaphorin3D (Sema3D), a class 3 semaphorin expressed by the spinal cord roof plate, is repulsive to peripheral, but not central, axons and may act to repel the peripheral branch out of the spinal cord. In contrast, transient axonal glycoprotein-1 (TAG-1), an Ig superfamily molecule located on R-B axons, is required for central axons to advance along their pathway, but it is not required for peripheral axon outgrowth or guidance.

\section{Materials and Methods}

Morpholino injection. Morpholino (MO) antisense oligos were synthesized by Gene Tools (Corvallis, OR). The MO sequences included the following: Sema3D translation-blocking MO (3DMO), 5' -CATGATGGACGAGGAGATTTCTGCA-3'; four mispair control, 5' -CATcATGcACGAGGAGATaTCTcCA-3'; Sema3D splice-blocking MO (3DMO2), 5'-CACATTCAGTCTGCAGCAAGAGAAA-3'; standard control, 5' CCTCTTACCTCAGTTACAATTTATA-3'; TAG-1 translation-blocking MO (TAGMO1), 5'-CCACACCCAGACCAGACACTTATTT-3'; TAG-1 splice-blocking MO (TAGMO2), 5'-TGACAAAAATGTGATGTGAGTACCG-3' . Morpholinos were diluted in Danieux buffer (Nasevicius and Ekker, 2000) and 0.1\% phenol red for injection. Approximately $1 \mathrm{nl}$ of a $500 \mu \mathrm{M}$ ( $1 \mathrm{~mm}$ for TAGMO2) solution was injected into embryos at the one- to two-cell stage.

Immunohistochemistry and in situ hybridization. Whole-mount immunohistochemistry was performed as described previously (Wolman et al., 2004). The zebrafish-derived neuronal marker (ZN-12) antibody (Zebrafish Resource Center, Eugene, OR) was used at a 1:250 dilution, and the anti-TAG-1 antibody (gift from Dr. Claudia Stuermer, University of Konstanz, Konstanz, Germany) was used at 1:500. Double-labeled embryos were imaged on a Zeiss (Oberkochen, Germany) Axiovert 100M confocal microscope with the Bio-Rad (Hercules, CA) 1024 Lasersharp Confocal software package. Step sizes were $0.5 \mu \mathrm{m}$. Whole-mount in situ hybridization was performed as described previously (Halloran et al., 1999).

Live imaging. Embryos were anesthetized in 0.02\% 3-amino benzoic acid ethylester (tricaine) and immobilized in 3\% methyl cellulose; a $0.2 \%$ solution of 1,1' -dioctadecyl-3,3,3',3' -tetramethylindocarbocyanine perchlorate (DiI; Invitrogen, San Diego, CA) was pressure-injected into one to three localized sites along the axis of the dorsal spinal cord. Embryos ranged in age from 19-23 h postfertilization (hpf) at the start of imaging and were imaged for $2-10 \mathrm{~h}$. For imaging, the embryos were mounted in $1 \%$ low-melting agarose in embryo medium (in mM: $5 \mathrm{NaCl}, 0.17 \mathrm{KCl}$,
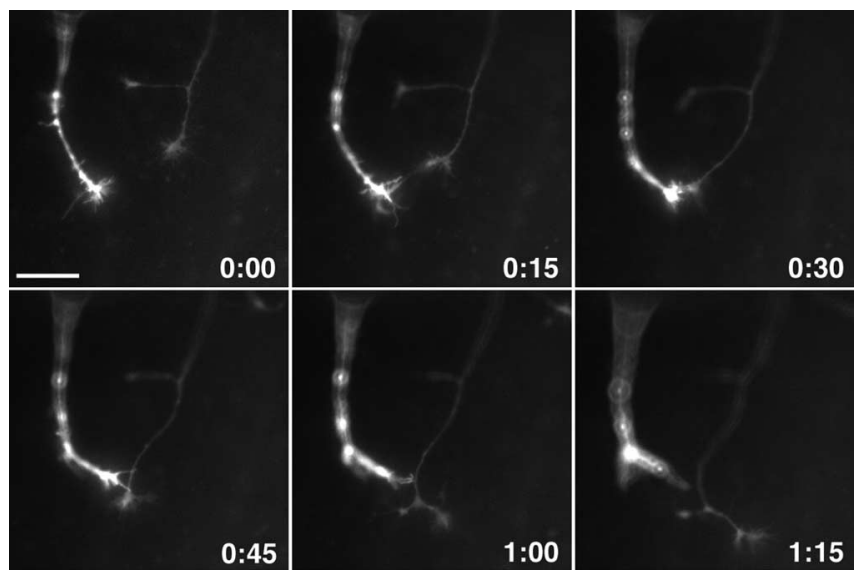

Figure 2. Peripheral R-B growth cones repel each other. Images are from time-lapse sequence showing mutual repulsion between two contacting peripheral growth cones. The right growth cone turns away while the left growth cone retracts. Scale bar, $20 \mu \mathrm{m}$.

$0.33 \mathrm{CaCl}_{2}, 0.33 \mathrm{MgSO}_{4}$ ) with $0.02 \%$ tricaine and 15 mM HEPES. Imaging was performed at $27-28^{\circ} \mathrm{C}$. Images were captured on a Nikon (Tokyo, Japan) E-600FN equipped with standard epifluorescence, a filter wheel, and a CoolSnap HQ camera (PhotoMetrics, Tucson, AZ). Images were captured every $1 \mathrm{~min}$, and exposure times were typically 100-600 ms. Images and movies were processed and analyzed with MetaMorph software (Universal Imaging Corporation, West Chester, PA).

Ectopic Sema3D expression. Approximately $1 \mathrm{nl}$ of a $50 \mu \mathrm{g} / \mathrm{ml}$ solution of DNA encoding a Sema3D-enhanced green fluorescent protein (EGFP) fusion protein driven by the zebrafish heat shock promoter 70 (hsp70) (Halloran et al., 2000) was injected into the blastomere of onecell stage embryos. At 15-16 hpf, the ectopic Sema3D-EGFP was heatinduced by placing embryos at $39^{\circ} \mathrm{C}$ for $1 \mathrm{~h}$. Embryos were allowed to develop for another $2-3 \mathrm{~h}$ at $28.5^{\circ} \mathrm{C}$ before $\mathrm{Dil}$ injection to label the R-B axons. For two-color imaging, the red (DiI) image and then the green (EGFP) image were captured in immediate succession at each time point. Images were captured every $2 \mathrm{~min}$.

Statistics. We used two-tailed Student's $t$ tests, assuming equal variances. Means are expressed \pm SEM. 


\section{Results}

Live imaging of R-B axon development $\mathrm{R}-\mathrm{B}$ cell bodies lie in two bilateral rows within the dorsal spinal cord on either side of the dorsal midline roof plate and extend central axons anteriorly and posteriorly, forming the dorsal longitudinal fasciculus (DLF). The central axons begin extending at $\sim 16 \mathrm{hpf}$ and pioneer the DLF in the spinal cord (Kuwada et al., 1990) (Fig. $1 A, B)$. Slightly later, $\sim 17-18 \mathrm{hpf}$, the peripheral axons begin to emerge. These axons exit the spinal cord, extend over the somites and under the epidermis, and arborize widely (Fig. 1C). Previous experiments using single-cell labeling showed that the peripheral axon arises as a branch directly from the central axon (Kuwada et al., 1990), a point confirmed below.

We used time-lapse imaging of living DiI-labeled R-B axons first to characterize normal development of the two axon branches and the motile behavior of their growth cones. The central axons generally extend steadily forward along straight trajectories with only occasional short pauses or small retractions. They advance at a net rate of $24.4 \pm 2.9 \mu \mathrm{m} / \mathrm{h}(n=20$ growth cones). The DLF is a relatively loose fasci-

cle, and, although not always bundled tightly, the central axons do grow along each other (Fig. $1 B$, inset). We imaged central axons extending in either the anterior or posterior direction and did not detect any differences in behavior or rate of outgrowth between the ascending or descending axons. Live imaging of newly forming peripheral branches confirms that they emerge directly from the central axon and extend out of the spinal cord (Fig. 1D-I) (supplemental movie 1, available at www.jneurosci.org as supplemental material). Peripheral growth cones in the epidermis also advance steadily, with very few pauses or retractions, in a general ventral direction and at an average rate of $23.9 \pm 1.5 \mu \mathrm{m} / \mathrm{h}(n=23)$. Peripheral axons branch several times to form a network that eventually covers the trunk. These secondary branches in the periphery typically form by bifurcation of the primary growth cone and steady advance of both branches. The formation of a widespread network of peripheral axons covering maximum epidermal surface area requires that the peripheral axons do not fasciculate along each other as the central axons do. In fact, our time-lapse imaging shows that the peripheral axons repel each other at contact. We analyzed 26 interactions between a peripheral R-B growth cone and another axon or growth cone. In 21 of these encounters, the growth cone retracted and/or turned away from the other axon/growth cone (Fig. 2 ). In the other five cases, the growth cone crossed over the axon, although in four of these cases, the growth cone first underwent multiple retractions or pauses before eventually crossing the axon. Repulsion between trigeminal axons and R-B axons also has been reported recently by others (Sagasti et al., 2005). These results suggest that mutual repulsion is a component of the mechanism by which R-B peripheral arbors spread widely in the skin. This result also demonstrates that a molecular difference between central and peripheral axons must exist from the early stages of development, which allows the central axons to extend along each other while causing the peripheral axons to be repelled by each other.

\section{Sema3D repels peripheral, but not central, $R-B$ axons}

We investigated the molecular mechanisms directing the central versus peripheral axon branches to behave differently. One potential guidance cue is Sema3D, a secreted member of the semaphorin family of axon guidance signals (Fiore and Puschel, 2003). We have shown previously that Sema3D is repulsive to retinal ganglion cell axons and midbrain axons, whereas it is attractive to telencephalic axons (Liu et al., 2004; Wolman et al., 2004). Sema3D is expressed in the spinal cord roof plate between the two rows of R-B cell bodies (Halloran et al., 1999) (Fig. $3 A, B$ ). The central R-B axons normally extend parallel to and close to the edge of the sema3D expression domain. In contrast, peripheral axons extend laterally away from the sema $3 D$ expression, suggesting that these axons may respond differently to Sema3D. We hypothesized that Sema3D may be repulsive to peripheral R-B axons and may function to repel them from the spinal cord.

We generated Sema3D-deficient embryos with antisense morpholino injection. We used a 3DMO that we have shown previously to be effective (Liu et al., 2004; Wolman et al., 2004), a 3DMO2 directed against the boundary of the fourth intron/fifth exon that causes a 118 nucleotide deletion in the sema domain (our unpublished observations), or a control morpholino with four bases mispaired from 3DMO (conMO). To test whether Sema3D influences the emergence of peripheral axons, we counted the number of peripheral axons extending from the central pathway out of the spinal cord. Embryos lacking Sema3D exhibited fewer peripheral axons exiting the spinal cord. ConMO-injected embryos $(n=48)$ had an average of $19.0 \pm 0.53$ peripheral axons exiting from segment levels 5-11, whereas 3DMO-injected embryos $(n=42)$ had an average of $11.4 \pm 0.65$, a highly significant difference ( $p=10^{-14}$; two-tailed Student's $t$ test) (Fig. 3C,D). Embryos injected with 3DMO2 $(n=16)$ had $12.2 \pm 0.49$ peripheral axons, also significantly different from controls $\left(p=10^{-8}\right)$. ConMO-, 3DMO-, and 3DMO2-injected 


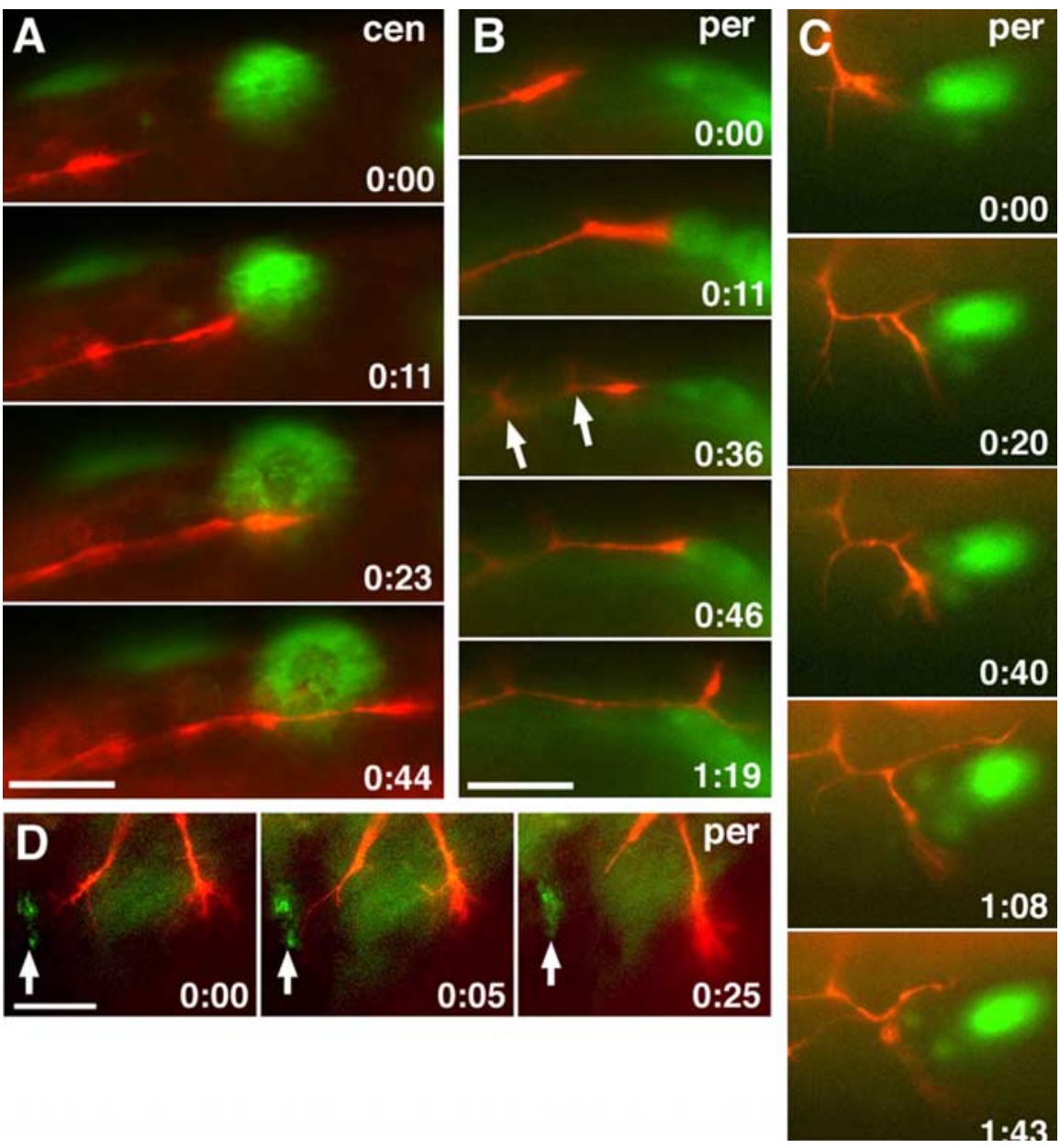

Figure 4. Peripheral, but not central, growth cones respond to ectopic Sema3D. A, Images from time-lapse sequence of R-B central axon (red) encountering and extending past an ectopic Sema3D-EGFP-expressing cell (green). $\boldsymbol{B}$ - $\boldsymbol{D}$, Images from timelapse sequences of peripheral growth cones contacting ectopic Sema3D-EGFP-expressing cells. $\boldsymbol{B}$, Growth cone retraction and concurrent back-branching (arrows). C, Growth cone branching at Sema3D cell and branches repelled by cell. D, Two branches of a peripheral axon, one of which contacts a Sema3D-expressing cell (arrow in each panel) and retracts. The larger green area is out-of-focus fluorescence. Scale bars, $20 \mu \mathrm{m}$.

embryos were age-matched by somite number. The axons that did emerge were shorter in Sema3D knock-down embryos (Fig. $3 C, D)$, suggesting that the difference may reflect a delay in peripheral axon outgrowth. However, there was no delay or detectable defect in the outgrowth of the central R-B axons. The central axons had extended and joined to form a continuous tract along the length of the trunk by $17 \mathrm{hpf}$ in both $3 \mathrm{DMO}$-injected $(n=20)$ and conMO-injected $(n=20)$ embryos (Fig. $3 E, F)$. These results suggest that Sema3D secreted from the spinal cord roof plate helps to propel the peripheral axons out of the spinal cord.

To determine whether Sema3D is indeed repulsive to R-B axons and whether central versus peripheral growth cones respond differently to Sema3D, we misexpressed Sema3D and imaged growth cones that were encountering ectopic Sema3D. We injected DNA encoding a Sema3D-EGFP fusion protein driven by the $h s p 70$ heat-inducible promoter (Halloran et al., 2000). Embryos were injected at the one-cell stage, and ectopic Sema3D-EGFP expression was heat-induced just before R-B axon outgrowth. This results in random mosaic ectopic expression of Sema3D-EGFP. For central R-B growth cone imaging, we selected embryos with ectopic Sema3D-expressing cells in the spinal cord along the path of a DiI-labeled axon. Central axons rarely showed a response to ectopic Sema3D. Of 17 central growth cones that were imaged, 13 did not respond in any obvious manner to the Sema3D-expressing cells (Fig. 4A). One growth cone retracted transiently and then grew around the Sema3D-expressing cell, two paused transiently and then grew over the cell, and one veered around a Sema3Dexpressing cell in its pathway without pausing or retracting. It is possible that ectopic Sema3D in the CNS is masked by nearby endogenous Sema3D. Nevertheless, these central growth cones extend steadily in the presence of Sema3D without retracting, suggesting that they are not repelled by it.

In contrast, peripheral growth cones displayed robust behavioral responses to ectopic Sema3D, including retraction, turning, and branching. Embryos with ectopic expression in the overlying epidermal cells or underlying somite cells along the pathway of the axon were selected for imaging. Of 25 growth cones that were imaged, 22 responded to ectopic Sema3D (Fig. 4B-D) (supplemental movies 2-4, available at www.jneurosci.org as supplemental material). Seven of these retracted at contact with the Sema3D-expressing cell and did not recover within at least $2 \mathrm{~h}$ (Fig. 4D) (supplemental movie 2, available at www.jneurosci.org as supplemental material). Four growth cones retracted transiently and then diverted their path to grow around the cell, one growth cone grew over the Sema3D-expressing cell after two transient retractions, five growth cones turned away from the Sema3Dexpressing cell without retracting, and five branched at contact with a Sema3Dexpressing cell without retraction or turning. In addition, branching frequently was seen in combination with the other behaviors. For example, the growth cone in Figure $4 B$ (supplemental movie 3, available at www.jneurosci.org as supplemental material) retracted at contact with the Sema3D cell and at the same time extended back branches off the axon. It then grew forward alongside the cell and branched away from the cell. Only three peripheral growth cones appeared to be unaffected by ectopic Sema3D, because they extended over the cell without pausing or retracting.

The behaviors that peripheral axons display when encountering ectopic Sema3D are very different from control behaviors. As noted in the previous section, peripheral growth cones in wildtype embryos extended steadily, and branches formed by smooth bifurcation of growth cones. We also analyzed 18 growth cones in wild-type embryos exposed to the same heat treatment used to induce ectopic Sema3D and scored the frequency of retractions lasting $>3 \mathrm{~min}$. These growth cones infrequently (eight retractions by four growth cones) underwent small retractions (10 $\mu \mathrm{m}$ or less) lasting 4-6 min each over a total image time of $>2 \mathrm{~h}$ for each growth cone. These results suggest that the more dramatic behaviors of growth cones contacting Sema3D are not typical or caused by heat treatment. Overall, our results suggest that 
Sema3D is repulsive to peripheral R-B axons and may induce branching while having little or no effect on central axons.

TAG-1 is required for central, but not peripheral, $\mathrm{R}-\mathrm{B}$ axon guidance

To gain additional insight into the mechanisms of R-B axon guidance, we also investigated the role of the Ig superfamily molecule TAG-1. TAG-1 is a glycosylphosphatidylinositol-linked (GPI-linked) membrane protein that is expressed by $\mathrm{R}-\mathrm{B}$ cells during the time of their axon outgrowth (Warren et al., 1999). TAG-1 can bind homophilically or heterophilically with other members of the Ig superfamily to mediate adhesion or signaling between cells or axons (Furley et al., 1990; Kuhn et al., 1991; Rader et al., 1993; Felsenfeld et al., 1994; Fitzli et al., 2000; Perrin et al., 2001; Pavlou et al., 2002; Karagogeos, 2003). We examined TAG-1 protein distribution on R-B axons by labeling with an anti-TAG-1 antibody (Lang et al., 2001). TAG-1 protein was expressed strongly on the central R-B axons at all stages that were examined, 20-27 hpf (Fig. 5A-D). The peripheral axons expressed TAG-1 protein when they initially extended (Fig. 5E, shown at $21 \mathrm{hpf}$ ); however, expression on peripheral axons was almost undetectable by $27 \mathrm{hpf}$ (Fig. $5 \mathrm{~F}-\mathrm{H}$ ).

We used morpholino knock-down of TAG-1 to test its function in R-B axon guidance. We used two independent morpholinos against TAG-1, one directed against the translation start (TAGMO1) and another against the boundary of exon 2/intron2 (TAGMO2) to block mRNA splicing. To verify their effectiveness at blocking TAG-1 protein, we injected embryos with TAGMO1, TAGMO2, or a standard conMO and labeled them with the anti-TAG-1 antibody. Both morpholinos caused a complete loss of detectable anti-TAG-1 immunolabeling ( $n>50 \mathrm{em}-$ bryos per group) (Fig. 5I,J) (data not shown). We examined the effects of TAG-1 knock-down first by antibody labeling of R-B neurons to analyze all R-B axons. In embryos lacking TAG-1, the central R-B axons were defasciculated, showed undulating trajectories, and appeared shorter compared with the conMO-injected embryos (Fig. $5 K-M$ ). In contrast, there was no detectable difference in the peripheral R-B axons after TAG-1 knock-down at a stage when TAG-1 still is expressed normally on the peripheral axons (Fig. $5 \mathrm{~N}, \mathrm{O}$ ). TAGMO1-injected embryos $(n=20)$ had an average of $18.9 \pm 0.58$ peripheral axons exiting from segment levels $5-11$, which was not significantly different from age-matched controls $(n=21 ; 18.0 \pm 0.55$ axons; $p=0.2$; two-tailed Student's $t$ test).

To determine the effect of TAG-1 knock-down on central and peripheral growth cone motility, we performed live imaging in TAG-1 knock-down embryos. The outgrowth of the central R-B growth cones was affected dramatically by the loss of TAG-1. These growth cones showed short periods of slow advance interspersed with periods of long pausing or retraction. They frequently remained in one location for hours without showing significant advance. They did, however, remain very active and motile, extending and retracting filopodia and lamellipodia (Fig. $6 A, B$ ) (supplemental movie 5, available at www.jneurosci.org as supplemental material). Thus, although protrusive activity was not affected, the overall advance of central growth cones was significantly slower after TAG-1 knock-down (net advance in TAGMO was $10.3 \pm 2.6 \mu \mathrm{m} / \mathrm{h}$ with $n=25$ vs $24.4 \pm 2.9 \mu \mathrm{m} / \mathrm{h}$ in control with $n=20$ ) (Fig. $6 E$ ). In contrast, the peripheral growth cones were unaffected by TAG-1 knock-down. Their appearance, behavior, and rate of advance were indistinguishable from wild- 


\section{central}

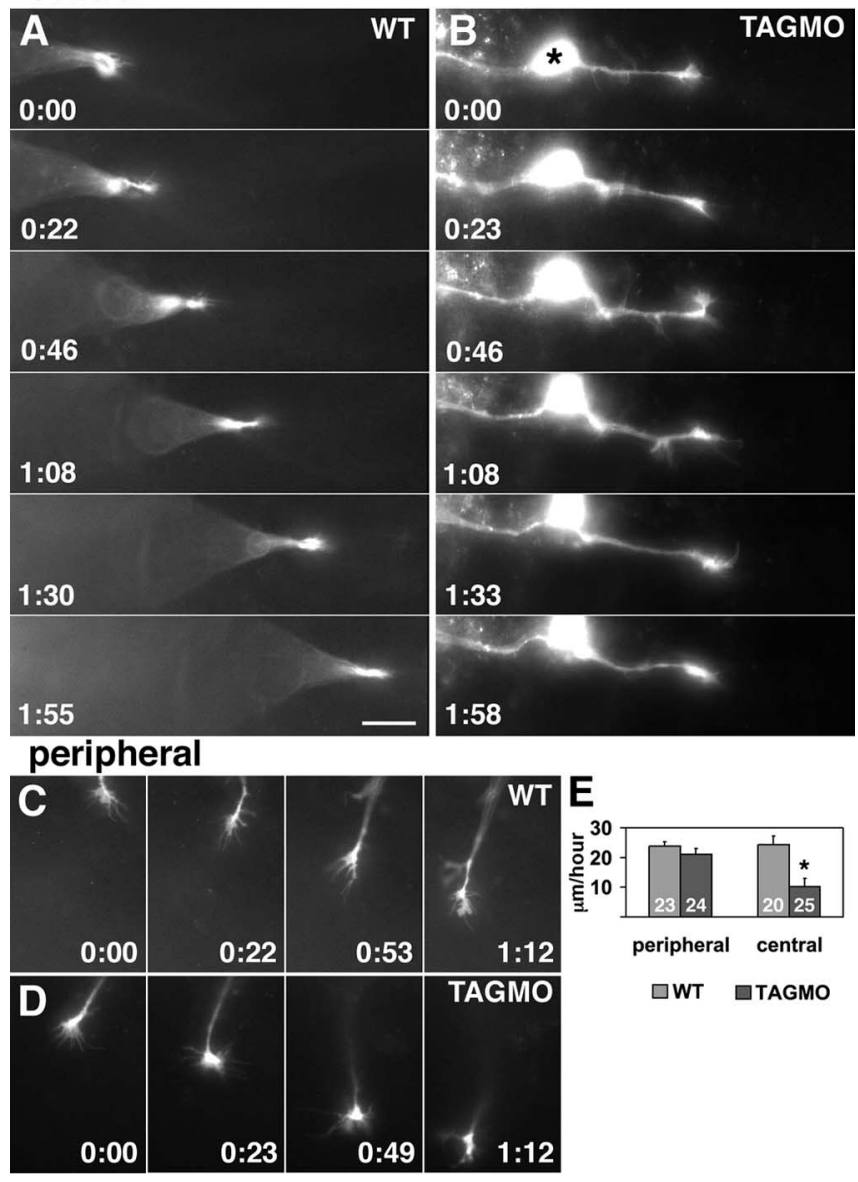

Figure 6. TAG-1 knock-down inhibits growth of central, but not peripheral, axons. $A$, Images from time-lapse sequence showing wild-type central growth cone advancing during $1 \mathrm{~h}, 55$ min. $\boldsymbol{B}$, Images of central growth cone in TAGMO-injected embryo showing no significant advance over the period of $1 \mathrm{~h}, 58 \mathrm{~min}$. The asterisk indicates cell body. $C, D$, Images of peripheral growth cone extending for $72 \mathrm{~min}$ in wild-type embryo (C) or TAGM0 embryo (D). E, Quantification of average growth rates. $n$ is indicated inside bars; error bars show SEM. *Significantly different from control ( $p=0.0008$; two-tailed $t$ test). Scale bar, $20 \mu \mathrm{m}$.

type growth cones (net advance in TAGMO was $21.1 \pm 1.9 \mu \mathrm{m} / \mathrm{h}$ with $n=24$ vs $23.9 \pm 1.5 \mu \mathrm{m} / \mathrm{h}$ in control with $n=23$ ) (Fig. $6 C-E)$.

\section{Discussion}

These studies reveal striking molecular and dynamic differences between axons from the same neuron, providing insight into how two axon branches from one cell can extend along different pathways to distinct targets. We have shown with in vivo live imaging that the growth cones of two R-B axon branches respond differently to an inhibitory cue, Sema3D, and have different requirements for a growth-promoting molecule, TAG-1.

\section{Sema3D role in $\mathrm{R}-\mathrm{B}$ axon development}

We found that Sema3D knock-down resulted in a significant reduction in the number of peripheral axons that extend from the central path out of the spinal cord. In addition, ectopic Sema3D expression elicited a repulsive response by peripheral growth cones but little or no response by central growth cones. These data suggest that the normal function of Sema3D may be to direct the peripheral branch away from the midline and out of the spinal cord. Interestingly, peripheral axons also frequently branched when contacting ectopic Sema3D. Sema3A and other repulsive cues have been shown to stimulate branches from retinal axons after growth cone collapse in vitro (Davenport et al., 1999; Campbell et al., 2001). However, Sema3A also can inhibit branching of cortical or hippocampal neurons in vitro (Bagnard et al., 1998; Bagri et al., 2003; Dent et al., 2004), suggesting that the particular effect of Sema3A on branching may depend on the specific neuronal type or on the environment. Our experiments show that, in an otherwise normal in vivo environment, ectopic Sema3D can stimulate branching by peripheral R-B growth cones. This also suggests a second possible role for Sema3D in stimulating the initial branching of the peripheral axons as they emerge from the spinal cord.

What is the molecular basis for the differential response to Sema3D? One possibility is that receptor components may be localized preferentially to the forming peripheral branch. Growth cones have been shown to regulate their receptors dynamically, with profound effects on their response to guidance cues (Dickson, 2002; van Horck et al., 2004). Receptors for class 3 semaphorins are composed of neuropilin and plexin components (Pasterkamp and Kolodkin, 2003). Several zebrafish neuropilins are expressed in the dorsal spinal cord (Bovenkamp et al., 2004; Yu et al., 2004); however, their protein localization is unknown. PlexinA4 is expressed in R-B cells, but a PlexinA4-EGFP fusion protein localizes to both peripheral and central R-B axons (Miyashita et al., 2004). An alternative possibility is that the central versus peripheral differences occur downstream of receptor activation. This appears to be the case for the differential effect of Sema3A on cortical axons versus dendrites, which is thought to be mediated by different levels of cGMP in axons and dendrites (Polleux et al., 2000). In the future, it will be interesting to explore the differences in receptor and downstream signaling components between central and peripheral R-B axons.

Sema3D likely acts together with other cues such as other roof plate repellents or attractants from the periphery to guide peripheral R-B branches. Secreted target-derived attractants have been shown to elicit side branches from cortical axons (Heffner et al., 1990). Loss of function of the Isl2 transcription factor, which is expressed in R-B cells, results in an elimination of peripheral R-B branches (Segawa et al., 2001). This suggests that Isl2 may control the expression of receptors or signaling components in R-B cells required to extend peripheral branches. The only signal downstream of Isl2 identified thus far is PlexinA4, which plays a role in the secondary branching of peripheral axons after they have extended into the skin but not in their initial formation (Miyashita et al., 2004). Plexins are known to act as semaphorin receptors. However, it is another guidance cue, Slit2, which is expressed in the somites (Yeo et al., 2001), that appears to be the ligand signaling via PlexinA4 to cause R-B axon branching (Miyashita et al., 2004). It is possible that Sema3D and Slit2 both act via PlexinA4 to help send the peripheral branches out of the spinal cord (Sema3D) and stimulate secondary arborization in the periphery (Slit2).

\section{TAG-1 is required for central R-B axon advance}

We have shown with live-imaging experiments that TAG-1 is required for the extension of central R-B axons but not for protrusive activity of their growth cones. These growth cones remain highly active after TAG-1 knock-down, extending and retracting numerous lamellipodia and filopodia. However, they make very little overall forward advance. This suggests that TAG-1 may regulate adhesions necessary for net advance of the growth cone. TAG-1 potentially could act via homophilic or heterophilic interactions with other Ig superfamily molecules. Some evidence 
suggests that heterophilic interactions are important. First, when central growth cones initially extend, they are not in contact with other TAG-1-expressing axons until they span the space between R-B cells. Second, when R-B cells were ablated from several segments in Japanese medaka fish, the axons from cells adjacent to the ablated area extended normally through the region devoid of axons, contacting only neuroepithelial endfeet (Kuwada, 1986). These experiments show that $\mathrm{R}-\mathrm{B}$ axons do not require other axons to extend and suggest that TAG-1 may interact heterophilically with cues present on the neuroepithelium. Although TAG-1 has been shown to bind homophilically in vitro (Rader et al., 1993; Pavlou et al., 2002; Karagogeos, 2003), thus far its functions in vivo are proposed to be via heterophilic interactions (Stoeckli and Landmesser, 1995; Fitzli et al., 2000; Perrin et al., 2001).

In contrast to the central axons, peripheral axons do not require TAG-1 to extend normally, demonstrating another molecular difference between the two branches. Presumably different adhesion-regulating molecules mediate peripheral axon advance. It is interesting that the peripheral branches avoid extending along the central pathway, although they express TAG-1 protein during the early stages of their outgrowth. One possibility is that another protein localized to the forming peripheral branch interferes with TAG-1 and negates attraction of the peripheral branch to the central pathway.

In conclusion, our experiments show that two axon branches from one neuron have separate guidance mechanisms and that their growth cones have distinct behavioral responses to manipulation of guidance cues. This work thus gives insight into the mechanisms by which different axons from the same neuron can grow to and innervate different targets. In addition, these studies provide a basis for future experiments to determine how axonal compartments are established.

\section{References}

Bagnard D, Lohrum M, Uziel D, Puschel AW, Bolz J (1998) Semaphorins act as attractive and repulsive guidance signals during the development of cortical projections. Curr Opin Neurobiol 8:587-592.

Bagri A, Cheng HJ, Yaron A, Pleasure SJ, Tessier-Lavigne M (2003) Stereotyped pruning of long hippocampal axon branches triggered by retraction inducers of the semaphorin family. Cell 113:285-299.

Becker T, Ostendorff HP, Bossenz M, Schluter A, Becker CG, Peirano RI, Bach I (2002) Multiple functions of LIM domain-binding CLIM/NLI/ Ldb cofactors during zebrafish development. Mech Dev 117:75-85.

Behar O, Golden JA, Mashimo H, Schoen FJ, Fishman MC (1996) Semaphorin III is needed for normal patterning and growth of nerves, bones, and heart. Proc Natl Acad Sci USA 93:11780-11785.

Bernhardt RR, Chitnis AB, Lindamer L, Kuwada JY (1990) Identification of spinal neurons in the embryonic and larval zebrafish. J Comp Neurol 302:603-616.

Bovenkamp DE, Goishi K, Bahary N, Davidson AJ, Zhou Y, Becker T, Becker CG, Zon LI, Klagsbrun M (2004) Expression and mapping of duplicate neuropilin-1 and neuropilin-2 genes in developing zebrafish. Gene Expr Patterns 4:361-370.

Campbell DS, Regan AG, Lopez JS, Tannahill D, Harris WA, Holt CE (2001) Semaphorin 3A elicits stage-dependent collapse, turning, and branching in Xenopus retinal growth cones. J Neurosci 21:8538-8547.

Clarke JD, Hayes BP, Hunt SP, Roberts A (1984) Sensory physiology, anatomy and immunohistochemistry of Rohon-Beard neurones in embryos of Xenopus laevis. J Physiol (Lond) 348:511-525.

Davenport RW, Thies E, Cohen ML (1999) Neuronal growth cone collapse triggers lateral extensions along trailing axons. Nat Neurosci [Erratum (1999) 2:485] 2:254-259.

Dent EW, Barnes AM, Tang F, Kalil K (2004) Netrin-1 and semaphorin 3A promote or inhibit cortical axon branching, respectively, by reorganization of the cytoskeleton. J Neurosci 24:3002-3012.
Dickson BJ (2002) Molecular mechanisms of axon guidance. Science [Erratum (2003) 299:515] 298:1959-1964.

Felsenfeld DP, Hynes MA, Skoler KM, Furley AJ, Jessell TM (1994) TAG-1 can mediate homophilic binding, but neurite outgrowth on TAG-1 requires an L1-like molecule and $\beta 1$ integrins. Neuron 12:675-690.

Fiore R, Puschel AW (2003) The function of semaphorins during nervous system development. Front Biosci 8:S484-S499.

Fitzli D, Stoeckli ET, Kunz S, Siribour K, Rader C, Kunz B, Kozlov SV, Buchstaller A, Lane RP, Suter DM, Dreyer WJ, Sonderegger P (2000) A direct interaction of axonin- 1 with $\mathrm{NgCAM}$-related cell adhesion molecule (NrCAM) results in guidance, but not growth, of commissural axons. J Cell Biol 149:951-968.

Furley AJ, Morton SB, Manalo D, Karagogeos D, Dodd J, Jessell TM (1990) The axonal glycoprotein TAG-1 is an immunoglobulin superfamily member with neurite outgrowth-promoting activity. Cell 61:157-170.

Furrer MP, Kim S, Wolf B, Chiba A (2003) Robo and Frazzled/DCC mediate dendritic guidance at the CNS midline. Nat Neurosci 6:223-230.

Halloran MC, Severance SM, Yee CS, Gemza DL, Raper JA, Kuwada JY (1999) Analysis of a zebrafish semaphorin reveals potential functions in vivo. Dev Dyn 214:13-25.

Halloran MC, Sato-Maeda M, Warren JT, Su F, Lele Z, Krone PH, Kuwada JY, Shoji W (2000) Laser-induced gene expression in specific cells of transgenic zebrafish. Development 127:1953-1960.

Heffner CD, Lumsden AG, O'Leary DD (1990) Target control of collateral extension and directional axon growth in the mammalian brain. Science 247:217-220.

Karagogeos D (2003) Neural GPI-anchored cell adhesion molecules. Front Biosci 8:S1304-S1320.

Kim S, Chiba A (2004) Dendritic guidance. Trends Neurosci 27:194-202.

Kuhn TB, Stoeckli ET, Condrau MA, Rathjen FG, Sonderegger P (1991) Neurite outgrowth on immobilized axonin-1 is mediated by a heterophilic interaction with L1(G4). J Cell Biol 115:1113-1126.

Kuwada JY (1986) Cell recognition by neuronal growth cones in a simple vertebrate embryo. Science 233:740-746.

Kuwada JY, Bernhardt RR, Nguyen N (1990) Development of spinal neurons and tracts in the zebrafish embryo. J Comp Neurol 302:617-628.

Lang DM, Warren Jr JT, Klisa C, Stuermer CA (2001) Topographic restriction of TAG-1 expression in the developing retinotectal pathway and target-dependent reexpression during axon regeneration. Mol Cell Neurosci 17:398-414.

Liu Y, Berndt J, Su F, Tawarayama H, Shoji W, Kuwada JY, Halloran MC (2004) Semaphorin3D guides retinal axons along the dorsoventral axis of the tectum. J Neurosci 24:310-318.

Miyashita T, Yeo SY, Hirate Y, Segawa H, Wada H, Little MH, Yamada T, Takahashi N, Okamoto H (2004) PlexinA4 is necessary as a downstream target of Islet2 to mediate Slit signaling for promotion of sensory axon branching. Development 131:3705-3715.

Nasevicius A, Ekker SC (2000) Effective targeted gene "knockdown" in zebrafish. Nat Genet 26:216-220.

Pasterkamp RJ, Kolodkin AL (2003) Semaphorin junction: making tracks toward neural connectivity. Curr Opin Neurobiol 13:79-89.

Pavlou O, Theodorakis K, Falk J, Kutsche M, Schachner M, Faivre-Sarrailh C, Karagogeos D (2002) Analysis of interactions of the adhesion molecule TAG-1 and its domains with other immunoglobulin superfamily members. Mol Cell Neurosci 20:367-381.

Perrin FE, Rathjen FG, Stoeckli ET (2001) Distinct subpopulations of sensory afferents require F11 or axonin-1 for growth to their target layers within the spinal cord of the chick. Neuron 30:707-723.

Polleux F, Morrow T, Ghosh A (2000) Semaphorin 3A is a chemoattractant for cortical apical dendrites. Nature 404:567-573.

Rader C, Stoeckli ET, Ziegler U, Osterwalder T, Kunz B, Sonderegger P (1993) Cell-cell adhesion by homophilic interaction of the neuronal recognition molecule axonin-1. Eur J Biochem 215:133-141.

Sagasti A, Guido MR, Raible DW, Schier AF (2005) Repulsive interactions shape the morphologies and functional arrangement of zebrafish peripheral sensory arbors. Curr Biol 15:804-814.

Segawa H, Miyashita T, Hirate Y, Higashijima S, Chino N, Uyemura K, Kikuchi Y, Okamoto H (2001) Functional repression of Islet-2 by disruption of complex with Ldb impairs peripheral axonal outgrowth in embryonic zebrafish. Neuron 30:423-436.

Stoeckli ET, Landmesser LT (1995) Axonin-1, Nr-CAM, and Ng-CAM play 
different roles in the in vivo guidance of chick commissural neurons. Neuron 14:1165-1179.

Taniguchi M, Yuasa S, Fujisawa H, Naruse I, Saga S, Mishina M, Yagi T (1997) Disruption of semaphorin III/D gene causes severe abnormality in peripheral nerve projection. Neuron 19:519-530.

van Horck FP, Weinl C, Holt CE (2004) Retinal axon guidance: novel mechanisms for steering. Curr Opin Neurobiol 14:61-66.

Wang J, Zugates CT, Liang IH, Lee CH, Lee T (2002) Drosophila DSCAM is required for divergent segregation of sister branches and suppresses ectopic bifurcation of axons. Neuron 33:559-571.

Warren Jr JT, Chandrasekhar A, Kanki JP, Rangarajan R, Furley AJ, Kuwada JY (1999) Molecular cloning and developmental expression of a zebrafish axonal glycoprotein similar to TAG-1. Mech Dev 80:197-201.
Wolman MA, Liu Y, Tawarayama H, Shoji W, Halloran MC (2004) Repulsion and attraction of axons by Semaphorin $3 \mathrm{D}$ are mediated by different neuropilins in vivo. J Neurosci 24:8428-8435.

Yaron A, Huang PH, Cheng HJ, Tessier-Lavigne M (2005) Differential requirement for Plexin-A3 and -A4 in mediating responses of sensory and sympathetic neurons to distinct class 3 semaphorins. Neuron 45:513-523.

Yeo SY, Little MH, Yamada T, Miyashita T, Halloran MC, Kuwada JY, Huh TL, Okamoto H (2001) Overexpression of a Slit homologue impairs convergent extension of the mesoderm and causes cyclopia in embryonic zebrafish. Dev Biol 230:1-17.

Yu HH, Houart C, Moens CB (2004) Cloning and embryonic expression of zebrafish neuropilin genes. Gene Expr Patterns 4:371-378. 